\title{
An Integrative Evolutionary Framework for Psychopathology
}

\author{
Marco Del Giudice \\ University of New Mexico \\ John D. Haltigan \\ University of Toronto
}

In press: Development and Psychopathology (accepted manuscript, June 2021)

Marco Del Giudice, Department of Psychology, University of New Mexico; John D. Haltigan, Department of Psychiatry, University of Toronto.

Address correspondence to Marco Del Giudice, Department of Psychology, University of New Mexico. Logan Hall, 2001 Redondo Dr. NE, Albuquerque, NM 87131, USA; email: marcodg@unm.edu 


\begin{abstract}
The field of psychopathology is in a transformative phase, and is witnessing a renewed surge of interest in theoretical models of mental disorders. While many interesting proposals are competing for attention in the literature, they tend to focus narrowly on the proximate level of analysis and lack a broader understanding of biological function. In this paper, we present an integrative framework for mental disorders built on concepts from life history theory, and describe a taxonomy of mental disorders based on its principles, the Fast-Slow-Defense model (FSD). The FSD integrates psychopathology with normative individual differences in personality and behavior, and allows researchers to draw principled distinctions between broad clusters of disorders, as well as identify functional subtypes within current diagnostic categories. Simulation work demonstrates that the model can explain the large-scale structure of comorbidity, including the apparent emergence of a general "p factor" of psychopathology. A life history approach also provides novel integrative insights into the role of environmental risk/protective factors and the developmental trajectories of various disorders.
\end{abstract}

Keywords: comorbidity; evolutionary psychiatry; evolutionary psychopathology; heterogeneity; life history strategies; $\mathrm{p}$ factor; transdiagnostic models. 
The field of psychopathology is in a transformative phase, as evidenced most clearly by the renewed surge of interest in theory and models. As the Diagnostic and Statistical Manual (DSM; American Psychiatric Association, 2013) ceases to work as a center of gravity for the discipline, the lack of a common framework becomes more apparent, and the need for innovative approaches grows more acute. Three currents stand out in today's literature. Dimensional transdiagnostic models (most notably the Hierarchical Taxonomy of Psychopathology or HiTOP; Conway et al., 2019; Kotov et al., 2017; Krueger et al., 2018) and network models (e.g., Borsboom \& Cramer, 2013; Borsboom et al., 2019) are largely inductive, driven by empirical patterns rather than theoretical concepts. On the other side are theory-first models that aim to explain mental disorders by appealing to general principles - such as predictive coding/active inference (e.g., Seth \& Friston, 2016), decision theory and reinforcement learning (e.g., Huys et al., 2015; Voon et al., 2017), or feedback control of goal-directed behavior in the tradition of cybernetics (DeYoung \& Krueger, 2018). Finally, bottom-up approaches such as the Research Domain Criteria (RDoC; Cuthbert \& Insel, 2013) focus on specific neural and cognitive mechanisms implicated in common disorders, with little consideration of broader conceptual issues (see Dalgeish et al., 2020; Wakefield, 2014).

To qualify as an integrative framework for the discipline, a candidate approach should meet four challenges: (a) explain large-scale patterns of comorbidity and overlap among disorders, at the phenotypic and genetic level; (b) address the mirror problem of heterogeneity within diagnostic categories and dimensions; (c) link the structure of pathological conditions ("kinds of disorders") to that of individual differences in personality and cognition ("kinds of people"); and (d) illuminate the developmental trajectories of mental disorders, and the interplay of risk and protective factors across the life course. While one can make progress in each of these areas from a purely mechanistic standpoint, we believe that successful integration requires a functional perspective on the mind and behavior, capable of explaining systems in terms of their reasons and purposes. Without doubt, current approaches such as cybernetic and active inference models embody important functional principles; but they usually stop at the proximate level of individual behavior, and fail to explicitly consider the ultimate source of function in living organisms - evolution by natural selection. Like the broader disciplines of psychology and medicine (Brüne \& Schiefenhovel, 2019; Buss, 2015; Stearns \& Medzhitov, 2016), psychiatry and psychopathology should embrace the evolutionary metatheory, and recast mental disorders within a broader, naturalistic understanding of function and dysfunction (Brüne, 2015; Brüne et al., 2012; Del Giudice, 2016a, 2018; Durisko et al., 2016; Keller, 2018; McGuire \& Troisi, 1998; Nesse, 2019; Nesse \& Jackson, 2006).

In this paper, we briefly review the principles of evolutionary medicine - the approach to medicine that views diseases and treatments through the lens of evolutionary biology, integrating the proximate and ultimate levels of analysis - and discuss some implications for the etiology of mental disorders. We then present an evolutionary framework for psychopathology based on the concept of life history strategies (Del Giudice, 2014, 2018). The framework aims to provide a conceptually unified answer to crucial questions of comorbidity, heterogeneity, normal variation, and development. The life history framework is the basis for a functional taxonomy of mental disorders, the Fast-Slow-Defense model (FSD). We illustrate the model by discussing the classification of autism and schizophrenia; in doing so, we present a selection of recent genomic 
evidence that was not available when the current version of the FSD model was published (Del Giudice, 2018). We also offer an updated overview of key similarities and differences between the FSD and HiTOP models, and some reflections on the meaning of the general factor of psychopathology ("p factor;" see Caspi \& Moffitt, 2018) from the standpoint of the life history framework.

\section{Evolution and the Etiology of Mental Disorders}

Our bodies and brains are the product of millions of years of natural selection - a process whose ultimate currency is the replication of genes across generations, as encapsulated by the biological concept of fitness (see Hunt \& Hodgson, 2010; West \& Gardner, 2013). Most evolved mechanisms exhibit universal, species-typical features as well as heritable individual variation, and interact with the organism's environment throughout development. Organisms are complex systems made up of myriad interacting parts, shaped and fine-tuned across countless generations. The central question of evolutionary medicine, then, is: why are organisms vulnerable to disease in the first place?

As it turns out, there are only a handful of general answers to this question (Nesse, 2005, 2019; see also Durisko et al., 2016). First, natural selection is slow, resulting in vulnerability to fast-evolving pathogens and mismatches between evolved mechanisms and novel environments. Second, selection is inherently limited in what it can accomplish (e.g., harmful mutations arise constantly and take time to be removed from the gene pool; see Keller, 2018), and must always work within numerous constraints and design trade-offs. Third, selection favors successful reproduction rather than health, well-being, or even survival per se. Many traits that enhance reproduction (or, more broadly, genetic replication) have significant health costs. Adaptive defenses - physiological ones like fever and behavioral ones like panic or disgust - are often aversive, and have the potential to become harmful or counterproductive. Trade-offs between health and fitness are amplified by the existence of evolutionary conflicts, not just between individuals with divergent genetic interests (e.g., parents vs. offspring) but also between multiple sets of genes within an individual (e.g., maternally vs. paternally inherited genes; Crespi, 2010).

These principles can be unpacked by considering whether undesirable conditions (i.e., "disorders" in the broad, nonspecific sense employed in psychiatry) originate from malfunctioning mechanisms (and thus qualify as narrow-sense disorders or "harmful dysfunctions;" Wakefield, 1992, 1999), or from mechanisms that are performing their proper evolved functions. In the latter case, one can ask whether the effects are biologically adaptive (fitness-enhancing) or maladaptive (fitness-reducing), both at the population and at the individual level. The resulting taxonomy is shown in Figure 1 (Del Giudice, 2018). While many mental disorders are likely to reflect harmful dysfunctions (caused by mutations, infection, social stressors, and so forth), others could be adaptive phenotypes that are mistaken for diseases because of their socially or emotionally aversive qualities (Nesse \& Jackson, 2006). In between these extremes, disorders can stem from evolutionary and developmental mismatches, or arise as maladaptive outcomes of evolved mechanisms that are generally adaptive (e.g., avoidance learning may lead to the onset of panic disorder). 


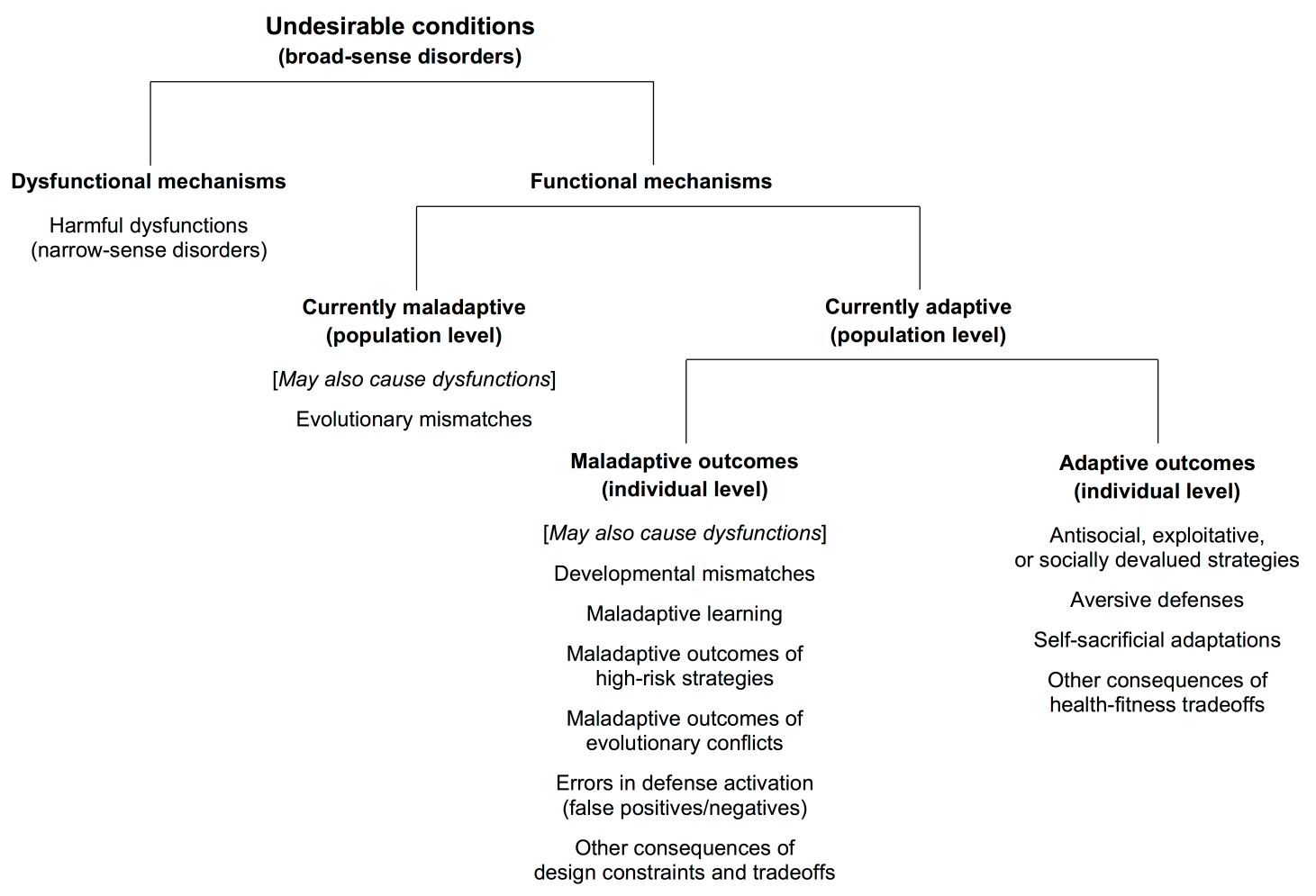

Figure 1. An evolutionary taxonomy of undesirable conditions (broad-sense disorders). Reproduced with permission from Del Giudice (2018).

The message of Figure 1 is that the biological roots of mental disorders are varied and complex, spanning the entire spectrum of adaptation and maladaptation (see also Cosmides \& Tooby, 1999; Syme \& Hagen, 2019). Accordingly, etiological theories that attempt to explain mental suffering with a few all-encompassing principles are destined to fail, or succeed only as partial explanations. A realistic approach to psychopathology requires a full arsenal of functional and mechanistic concepts, and the flexibility to address each condition on its own terms. The challenge, then, is to build a framework versatile enough to accommodate a diversity of specific models, but capable of bringing coherence to the field and fostering integration across multiple levels of explanation.

\section{The Life History Framework}

\section{Life History Strategies and the Fast-Slow Paradigm}

Life history theory is a branch of theoretical biology that describes how organisms evolve to adaptively allocate their resources (e.g., energy, time) to multiple components of fitness such as growth, survival, and reproduction (Roff, 2002; Stearns, 1992; for accessible overviews see Del Giudice et al., 2015; Ellis et al., 2009). At the most abstract and general level, an organism's life history strategy can be summarized by a few basic traits such as age at first 
reproduction, age-specific mortality, and age-specific fertility. These traits are the outcomes of a sequence of allocations to multiple fitness-relevant tasks; and because resources are necessarily limited, trade-offs arise so that maximizing one component of fitness comes at a cost for other, equally important components.

Three of the most important life history trade-offs are those between current and future reproduction, between the quality and quantity of offspring, and-for sexually reproducing species - between investment in mating and investment in parenting. The resolution of these trade-offs is determined by a combination of behaviors such as mating, pair-bonding, parental care, and aggression; physiological mechanisms such as metabolic regulation, immunity, and sexual maturation; and aspects of morphology such as adult body size and muscle mass. At the level of individual organisms, then, life history strategies are expressed as co-adapted suites of behavioral, physiological, and morphological traits (Braendle et al., 2011). Coordination among traits is often achieved through endocrine systems, such as the hypothalamic-pituitary-gonadal and hypothalamic-pituitary-adrenal axes. Hormones regulate the activity of multiple organs (including the brain), and can integrate genetic variation with information from the environment, thus providing a potential mechanism for the expression of developmental plasticity (Del Giudice, 2020; Vitousek \& Schoenle, 2019).

If one compares life history traits across multiple species, it becomes apparent that species can be arranged along a robust axis of variation known as the fast-slow continuum. "Fast" species show high mortality, early maturation and reproduction, and (in mammals and birds) high fertility, whereas "slow" species mature later, live longer, and tend to produce few offspring (see Del Giudice, 2020; Healy et al., 2019). Researchers in biology, psychology, and anthropology have extended the concept of a fast-slow continuum to describe variation among individuals within the same species, not just at the level of basic life history traits such as age at reproduction but also at the level of behavior and physiology. The goal of the "fast-slow paradigm" of individual differences (Del Giudice, 2020) is to account for patterns of covariation among traits by linking them to life history trade-offs. In this perspective, the fast-slow continuum is the broadest, most general level of functional description of individual differences; for this reason, it can be conceptually and heuristically useful even if it accounts for a limited proportion of variation.

The fast-slow paradigm is currently at the center of a spirited debate among proponents, critics, and reformers; for an overview see Nettle \& Frankenhuis (2019, 2020), Zietsch and Sidari (2020), Galipaud \& Kokko (2020), and Del Giudice (2020). Although the existence of a fast-slow continuum across species does not entail that the same pattern will necessarily be observed within a single species, the two levels are connected by basic life history trade-offs, such as those between current and future reproduction and mating versus parenting. Because these trade-offs are pervasive and functionally connected to one another (e.g., later reproduction should generally increase the potential for high-quality parental investment), it is reasonable to use the fast-slow continuum as a broad-band heuristic, even if within-species patterns do not precisely mirror their between-species counterparts (for in-depth discussion of these issues see Del Giudice, 2020). While the fast-slow continuum per se is an empirical generalization, life history trade-offs provide a theoretical basis for understanding how behavioral traits shift allocations toward different components of fitness (e.g., by promoting earlier vs. later 
reproduction and higher vs. lower mortality risk). Individual differences in life history-related traits within a species or population can be potentially maintained by a number of processes, including frequency-dependent selection and fluctuations in population density and sex ratios (see Del Giudice, 2012; Wright et al., 2019).

Behavioral traits can be linked to the fast-slow continuum to the extent that they predict the timing of sexual development and reproduction, patterns of mortality, and investment in mating versus parenting. In humans, the core cluster of "fast" life history-related traits comprises impulsivity, present orientation, risk-taking, and sensation seeking; low levels of conscientiousness and honesty-humility (Ashton \& Lee, 2008); precocious sexuality; preferences for uncommitted sex with multiple partners (unrestricted sociosexuality); unstable romantic attachments and reduced couple stability; and low sensitivity to moral and sexual disgust. All these traits correlate with one another, and predict life history-relevant outcomes such as larger numbers of sexual partners, earlier reproduction, and higher mortality. Other traits such as agreeableness and openness to experience show more complex patterns of association with the same outcomes, and can be linked to alternative "profiles" within fast and slow strategies (for a detailed account see Del Giudice, 2018). More speculatively, the proposed neurobiological correlates of life history strategies include serotonergic and oxytocinergic activity, sex hormone levels across development, and physiological patterns of stress reactivity (Del Giudice, 2018).

Fast behavioral traits are consistently associated with exposure early environmental adversity (e.g., family stress, maltreatment, trauma). A common assumption in the literature is that life history strategies show adaptive plasticity, consistent with the notion that faster strategies are maximally adaptive in harsh and/or unpredictable conditions (Belsky et al., 1991; Ellis et al., 2009; see Del Giudice et al., 2015). On the face of it, this assumption is not easy to reconcile with the findings of behavior genetics, which for most psychological traits seem to indicate a small or even negligible role of shared environmental factors (i.e., those that make siblings within the same family more similar to one another; Knopik et al., 2017; Zietsch \& Sidari, 2019). To begin, there are some important exceptions to the general rule, most notably antisocial behavior and substance use (which show a substantial shared environmental contribution of up to $20 \%$ of the variance; Kendler et al., 2019; Rhee \& Waldman, 2002). One should also note that quantifying shared environmental effects in terms of the proportion of variance they explain — as is customary in behavior genetics — can lead to dramatically underestimate their contribution relative to that of genetic and nonshared environmental effects (Del Giudice, 2021). An especially intriguing possibility is that the effects of the shared environment are partly masked by genotype-environment interactions, particularly if they involve differential susceptibility to both positive and negative experiences (see Belsky \& Pluess, 2016; Del Giudice, 2016b). However, it is fair to say that the causal role of early experiences in development remains poorly understood in many respects (e.g., Del Giudice, 2020; Ellis \& Del Giudice, 2019; Fraley et al., 2013).

\section{From Life History Strategies to Psychopathology}

The core proposition of the life history framework is that the risk for different types of mental disorders is partly dependent on broader patterns of individual differences, which in turn can be understood functionally by mapping them onto the fast-slow continuum. Individual 
differences on the fast-slow continuum pave the way for the development of psychopathology, increasing the risk for some symptoms and disorders and reducing the risk for others. The specific connections can take various forms, consistent with the multiple etiological pathways summarized in Figure 1. For example, adaptive life history-related traits may be regarded as pathological; traits that are adaptive on average may be expressed at maladaptive levels, or occasionally lead to undesirable outcomes; and different constellations of traits may increase the vulnerability to specific kinds of dysfunctions (Del Giudice, 2014, 2018). An important qualification is that, when diagnosable conditions are associated with significant cognitive impairment (e.g., schizophrenia), life history markers may be expressed more clearly in people with subclinical forms of the disorder (e.g., schizotypy) or in the patients' unaffected relatives.

The idea that some clinical conditions can be linked to fast strategies is not new (e.g., Belsky et al., 1991; Brüne et al., 2010; Mealey, 1995; Salmon et al., 2009). The life history framework extends this notion to slow strategies (marked by traits such as heightened selfcontrol and restricted sexual/mating behaviors), and provides a reasoned set of traits that can be used as convergent markers of fast versus slow strategies. In addition to the fast-slow axis, the framework includes another, largely independent axis for disorders whose primary symptoms reflect the intense and prolonged activation of defensive (i.e., self-protective) mechanisms such as anxiety, fear, panic, and sadness/depression. Defense activation symptoms may occur at both end of the fast-slow continuum for different functional reasons, although there are theoretical grounds to predict that they will be more strongly associated with fast traits (see Del Giudice, 2018).

The life history framework can be applied with two complementary objectives. The first is to map the large-scale structure of psychopathology, by describing broad clusters of disorders that share common functional correlates from a life history perspective (and hence should exhibit high comorbidity and familiarity). The second is to identify heterogeneous subtypes within existing diagnostic categories - e.g., by distinguishing between "slow" and "fast" variants of eating disorders with different personality/motivational correlates and constellations of comorbidity. The intention is not to replace existing evolutionary and etiological models of particular disorders, but to organize them into a coherent picture and provide the basis for a functional taxonomy. Of course, there are other functional principles that might be used as starting points; for example, one could attempt to map disorders on distinct motivational domains such as attachment and status competition (e.g., McGuire \& Troisi, 1998). Alternative taxonomies may be more or less useful depending on one's goals, and offer complementary insights into the nature of psychopathology.

An important advantage of a life history perspective is that it provides insights into the developmental patterns of different types of disorders, and a principled basis to reason about risk factors and epidemiological patterns. For instance, one can predict that disorders linked to fast traits will be more prevalent in conditions of high stress and adversity, whereas their slow counterpart should be more common in safe, stable environments that are usually regarded as protective. This may contribute in explaining the counterintuitive "J-shaped" relation that is often observed between adversity and psychological distress (Seery, 2011; Seery et al., 2013). Another prediction is that the onset of disorders linked to fast traits should peak between middle childhood and adolescence when they are functionally associated with social competition, and 
between adolescence and young adulthood when they are associated with mating and courtship. Additional refinements of the framework deal with patterns of sex differences in the risk for alternative types and subtypes of conditions (see Del Giudice, 2018).

\section{The FSD Model}

By applying the criteria described in the previous section, it is possible to classify psychopathological conditions and their subtypes into three broad categories of fast spectrum or F-type disorders, slow spectrum or S-type disorders, and defense activation or D-type disorders, based on their empirical patterns of associations with life history-related traits and outcomes. A residual category of $O$-type disorders (for "other") comprises conditions that seem to lack specific functional associations with life history-related traits or the activation of defensive mechanisms. The current version of the FSD model (as presented in Del Giudice, 2018) is summarized in Figure 2. Note that the model is still in development: some of the proposed classifications are admittedly tentative, and the coverage is wide but still incomplete (e.g., the current taxonomy does not cover substance use disorders, sexual dysfunctions, and paraphilias).

F-type conditions include disorders marked by disruptive and antisocial behaviors, including the DSM categories of conduct disorder (CD) and oppositional-defiant disorder (ODD); most instances of schizophrenia spectrum disorders (SSDs); a high-frequency subtype of bipolar disorders (F-BDs); a high-frequency subtype of attention-deficit/hyperactivity disorder, associated with conduct/antisocial behaviors and psychosis risk (F-ADHD); a subtype of eating disorders marked by high impulsivity and neuroticism, with a prevalence of bulimic symptoms (F-EDs); and personality disorders marked by high antagonism, disinhibition, or psychoticismnotably borderline, narcissistic, antisocial, and schizotypal personality disorders (BPD, NPD, ASPD, SPD). Crucially, the FSD model does not assume the validity of DSM categories, but employs them pragmatically (and provisionally) with the understanding that they may have to be modified or redefined in the future. For example, the definition and classification of personality disorders is manifestly problematic, schizophrenia and bipolar disorders show considerable functional overlap, and what are currently described as "subtypes of ADHD" may be best assimilated into other clinical entities (see Del Giudice, 2018 for details). The FSD model is intended as an initial step on the long road toward a fully evolutionary nosology of mental disorders.

S-type conditions include a mostly high-functioning subtype of autism spectrum disorders (S-ASD); a low-frequency subtype of bipolar disorders (S-BDs); a low-frequency subtype of ADHD that overlaps with autism (S-ADHD); a subtype of obsessive-compulsive disorder, primarily marked by feelings of incompleteness/imperfection and overlapping with autism (S-OCD); a subtype of eating disorders characterized by high conscientiousness, with high- and low-neuroticism variants (S-EDs); and personality disorders marked by elevated conscientiousness and/or agreeableness, most notably obsessive-compulsive personality disorder (OCPD).

D-type disorders include depression, generalized anxiety disorder (GAD), post-traumatic stress disorder (PTSD), social anxiety disorder (SAD) and avoidant personality disorder (APD), phobias, panic, and a subtype of OCD in which symptoms are primarily motivated by harm 
prevention (D-OCD). Finally, O-type conditions include severe forms of autism without functional links to the fast-slow continuum (O-ASD), and a subtype of ADHD mainly characterized by generalized cognitive deficits (O-ADHD).

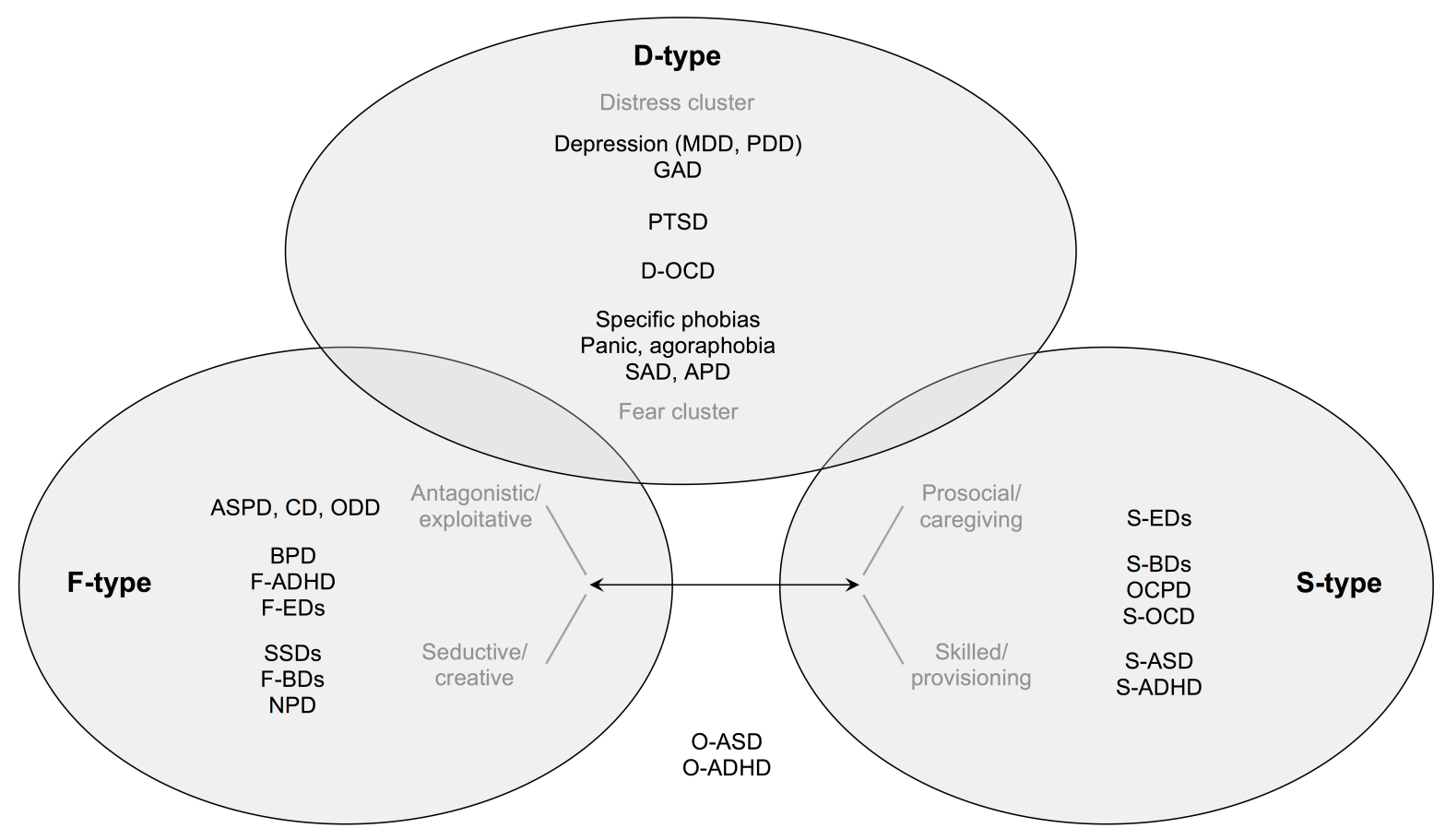

Figure 2. Classification of common DSM conditions as fast spectrum (F-type), slow spectrum (S-type), and defense activation (D-type) disorders in the current version of the FSD model. Conditions that fall outside these three categories (O-type) are shown at the bottom of the figure. $\mathrm{ADHD}=$ attention-deficit/hyperactivity disorder. $\mathrm{APD}=$ avoidant personality disorder. ASD $=$ autism spectrum disorder. ASPD $=$ antisocial personality disorder. $\mathrm{BDs}=$ bipolar disorders. $\mathrm{BPD}=$ borderline personality disorder. $\mathrm{CD}=$ conduct disorder. $\mathrm{EDs}=$ eating disorders. $\mathrm{GAD}=$ generalized anxiety disorder. MDD = major depressive disorder. $\mathrm{NPD}=$ narcissistic personality disorder. $\mathrm{OCD}=$ obsessive-compulsive disorder. $\mathrm{OCPD}=$ obsessive-compulsive personality disorder. $\mathrm{ODD}=$ oppositional-defiant disorder. $\mathrm{PDD}=$ persistent depressive disorder. $\mathrm{PTSD}=$ posttraumatic stress disorder. $\mathrm{SAD}=$ social anxiety disorder. SSDs = schizophrenia spectrum disorders. Reproduced with permission from Del Giudice (2018).

Relative to the DSM, the FSD model with its three main clusters stands out as a "lumping" taxonomy; at the same time, it involves a fair amount of "splitting," as several diagnostic categories - autism, ADHD, bipolar disorders, eating disorders, and OCD - are cleaved into functionally distinct subtypes. It is important to stress that the organization of disorders into three functional clusters is fully consistent with the idea that the disorders themselves are, for the most part, extremes of continuous distributions of symptoms rather than discrete natural categories or taxa (e.g., Haslam et al., 2012, 2020; see below for some likely exceptions). Thus, the F, S, and D groupings can equivalently be described as "categories", "clusters", or "spectra". 


\section{An Illustration: Autism and Schizophrenia}

We now illustrate the model by presenting the FSD classification of conditions in the spectra of autism (ASD) and schizophrenia (SSDs). For reasons of space, we provide only a brief outline; for a thorough discussion (including a review of the genetic, neurobiological, and developmental evidence), see Del Giudice (2018). In many respects, the FSD classification embraces the diametrical model of autism and psychosis (Crespi \& Badcock, 2008; Crespi, 2011), according to which ASD and psychosis-spectrum disorders (such as schizophrenia and bipolar disorders) lie at the opposite ends of a distribution of cognitive traits that trade off against one another-hyper-mechanistic cognition, low imagination, restricted attention, and enhanced visuospatial skills in autism versus hyper-mentalistic cognition, diffuse attention, high imagination, and poor visuospatial skills in psychosis. (Note that, in this context, "imagination" refers mainly to fantasy-oriented, divergent thinking with a strong affective and social component, which is more characteristic of artistic creativity. Thus, this term does not encompass the systematic forms of thinking and problem-solving that are more characteristic of scientific and technical creativity.) The diametrical phenotypes associated with autism and psychosis seem to partly reflect the diametrical action of maternally vs. paternally inherited imprinted genes (Crespi, 2019).

In the FSD model, SSDs are classified as F-type conditions, spanning the range from potentially adaptive phenotypes (mild schizotypal/schizoaffective forms, especially positive symptoms such as delusional/paranoid ideation and hallucinations) to frankly maladaptive dysfunctions (severe schizophrenia, especially early-onset presentations with a prevalence of negative symptoms). The behavioral and personality correlates of positive schizotypy include impulsivity, sensation seeking, risk-taking, and unrestricted sociosexuality, as well as reduced moral/sexual disgust (reviewed in Del Giudice, 2018). Crucially, genomic studies corroborate these behavioral findings: schizophrenia shows positive genetic correlations with risk-taking (Linnér et al., 2018), and there is evidence that the genetic risk for schizophrenia predicts earlier age at first intercourse and first birth (the latter with indications of a U-shaped relationship), as well as larger numbers of sexual partners (Lawn et al., 2019; Ni et al., 2019).

These data support an F-type classification, and are consistent with the hypothesis that sexual selection (for example via enhanced creativity and courtship skills) has contributed to the maintenance of schizotypal traits in human populations (Del Giudice, 2017; Nettle, 2001; Shaner et al., 2004; see also Keller, 2018). From this perspective, the low fertility of patients with schizophrenia is due to the fact that diagnosable schizophrenia is a dysfunctional outcome, arising from the interaction between a potentially adaptive predisposition (schizotypy) and severe environmental and/or genetic disruptions (e.g., infections, deleterious mutations; see Del Giudice, 2017). Many of the same considerations apply equally well to bipolar disorders, which show strong genetic and familial overlap with schizophrenia. However, there are indications that, in a minority of cases, bipolar symptoms may arise in the context of slow life history strategies (Del Giudice, 2018).

The FSD model distinguishes between two functionally independent subtypes of autism: a mostly high-functioning subtype (S-ASD), with high familiarity for autistic-like traits, a large contribution of common alleles, and a strongly male-biased prevalence; and a mostly low- 
functioning subtype (O-ASD) with high risk of intellectual disability, a large contribution of rare deleterious genetic mutations, and a more balanced prevalence between the sexes (Del Giudice, 2018). Consistent with an S-type classification, the risk for high-functioning ASD - but not for ASD with intellectual disability - seems to be associated with higher socioeconomic status (reviewed in Del Giudice, 2018). Autistic-like traits in the non-clinical population correlate with low impulsivity, risk aversion, low sensation seeking, restricted sociosexuality, as well as stable and committed couple relationships (Del Giudice et al., 2010, 2014). There is a negative genetic correlation between ASD and risk-taking (Linnér et al., 2018), and genetic risk for autism seems to predict delayed intercourse and later first birth (Ni et al., 2019). Similar to schizophrenia, sexual selection may have played a role in the evolution of autistic-like traits, but specifically in the context of long-term mating and extended parental investment (Del Giudice et al., 2010).

Note that the FSD model does not account for the specific etiology and evolutionary history of autism and schizophrenia, which is a task for narrower models of these disorders. Instead, by linking these disorders to broader constellations of traits, the model contributes to explain their patterns of comorbidity (Figure 2), make sense of their epidemiological and developmental features (e.g., age of onset, associations with socioeconomic status, effects of parental age at conception, role of deleterious mutations; see Del Giudice, 2018), and identify meaningful subtypes within extant diagnostic categories.

If the FSD classification is broadly correct, extant genomic studies of ASD likely include a mixture of functionally distinct conditions, and may hide as much as they reveal. For example, most studies detect a small, positive genetic correlation between ASD and schizophrenia (e.g., Grove et al., 2019; Lee et al., 2019; Warrier et al., 2019). This could be due to nonspecific factors in addition to diagnostic confusion, particularly in children (Crespi, 2011, 2020). A recent genomic study supports the idea that polygenic risk for ASD reflects two distinct genetic signatures, with different patterns of correlations with other traits and demographic variables (Zhang et al., 2020). By applying principal component analysis to polygenic risk scores (mainly based on common alleles), Selzam and colleagues (2018) found that genetic scores for both autism and schizophrenia loaded on a general first component, which they interpreted as a genetic "p factor" (see below); but after the first two components were rotated, autism and schizophrenia ended up loading on different components. This pattern mirrors the phenotypic distribution of autistic-like and schizotypal traits, and is compatible with a diametrical model of the two disorders (standard rotation algorithms tend to break bipolar constructs apart into two separate factors/components; see Del Giudice, 2020; Del Giudice et al., 2014).

\section{FSD vs. HiTOP: A Comparison}

Among current models of psychopathology, the HiTOP offers the most detailed and mature alternative to the DSM taxonomy, built on more than three decades of research in the transdiagnostic tradition (Conway et al., 2019; Kotov et al., 2017; Krueger et al., 2018). The HiTOP is similar to the FSD model in its broad and integrative scope, focus on transdiagnostic dimensions, and explicit connection with normal variation in personality. However, and in stark contrast with the FSD model, the approach to classification of the HiTOP is fundamentally atheoretical, and mainly driven by empirical patterns of similarities and correlations among symptoms and/or syndromes (Dalgeish et al., 2020; Haeffel et al., 2021). Hence, the dimensions 
or spectra identified in the HiTOP (and earlier transdiagnostic models) cut across existing diagnostic categories, but currently cannot be used to draw distinctions between conditions that share similar constellations of symptoms for functionally different reasons (see also Haeffel et al., 2021). One consequence is that DSM categories (which are not themselves part of the HiTOP taxonomy) are usually assigned to a particular HiTOP spectrum (e.g., externalizing for ADHD, internalizing for eating disorders; see Kotov et al., 2017). The exceptions are so-called "interstitial" conditions that are defined by multiple dimensions at once. For example, in the HiTOP model the symptoms of BPD are interstitial between the internalizing and externalizing dimensions, whereas bipolar symptoms are interstitial between internalizing and thought disorders (see Kotov et al., 2017). However, interstitial placement of symptoms or disorders does not correspond to functional subtypes; instead, the standard interpretation is that different components of the disorder may relate to different clinical spectra (e.g., see the discussion of BPD in Krueger et al., 2021).

In total, the transdiagnostic approach has helped describe large-scale patterns of comorbidity by subsuming multiple types of symptoms and disorders under broader clinical dimensions; but so far, it has provided limited insight into the problem of heterogeneity within existing categories and symptom dimensions. To illustrate, ADHD is widely recognized as a highly heterogeneous diagnostic label (see Del Giudice, 2018). At the genetic level, ADHD overlaps with both ASD and psychosis, in addition to conduct and antisocial symptoms (Lee et al. 2019; Linnér et al., 2020; Solberg et al., 2019; Warrier et al. 2019; Zhang et al. 2020). Likewise, relatives of ADHD patients are at heightened risk for disorders in both the autistic and psychotic spectrum (e.g., Larsson et al., 2013; Musser et al., 2014). However, the current HiTOP model unambiguously locates ADHD symptoms and related traits (such as "distractibility" and "impatient urgency") within the externalizing spectrum (Krueger et al., 2021; Conway et al., 2019). In principle, the evidence of overlap with psychosis could be absorbed in the HiTOP model with an "interstitial" placement of ADHD, with contributions from both the externalizing and thought disorder dimensions; alternatively, the associations with autism and psychosis could be explained as a downstream consequence of a general " $p$ factor" at the top of the hierarchy (see below). In contrast, the FSD model suggests that the DSM category of ADHD should be split into three subtypes (F-ADHD, S-ADHD, O-ADHD), with similar constellations of symptoms but different functional underpinnings and patterns of comorbidity with life history traits. As a second example, consider the classification of OCD and its symptoms; In the current HiTOP model, obsessions and compulsions are located within the internalizing spectrum (Conway et al., 2019). In the FSD model, obsessions and compulsions can arise from two fundamentally distinct processes: (a) a hyperactivated harm prevention mechanism (D-type) and (b) a heightened need for order and predictability, usually associated with obsessive, perfectionistic, and autistic-like personality traits (S-type).

Both the HiTOP and FSD models permit the possibility that some disorders may be taxonic rather than fully dimensional (see Kotov et al., 2017). Nonetheless, there is a difference in emphasis between the two approaches: proponents of the HiTOP model tend to make fairly strong assumptions of dimensionality based on the results of taxometric studies, whereas the FSD model is more agnostic in this regard. On the one hand, even if certain conditions result from the action of qualitatively distinct etiological processes, this may or may not give rise to the statistical patterns of discontinuity targeted by taxometric methods. (Stated differently, absence 
of detectable taxonicity at the statistical level does not necessarily imply that the same is true at the etiological level; see also Kendler, 2018). On the other hand, we view the taxometric evidence as less clear-cut than it is sometimes portrayed. For example, there is some support for the idea that severe ASD is categorically distinct from milder forms of the disorder (consistent with the idea of multiple subtypes with different functional underpinnings), and the evidence regarding schizotypy and SSDs is still mixed (Coghill \& Sonuga-Barke, 2012; Haslam et al., 2012, 2020). In some cases, the resolution at which the symptoms are parsed makes a difference; although the taxometric evidence in relation to the broad category of eating disorders is mixed (Haslam et al., 2020), results from a number of studies indicate that bingeing is probably taxonic, whereas food restriction is not (Hilbert et al. 2011; Thomas et al. 2015; Williamson et al. 2005; Zheng et al. 2019).

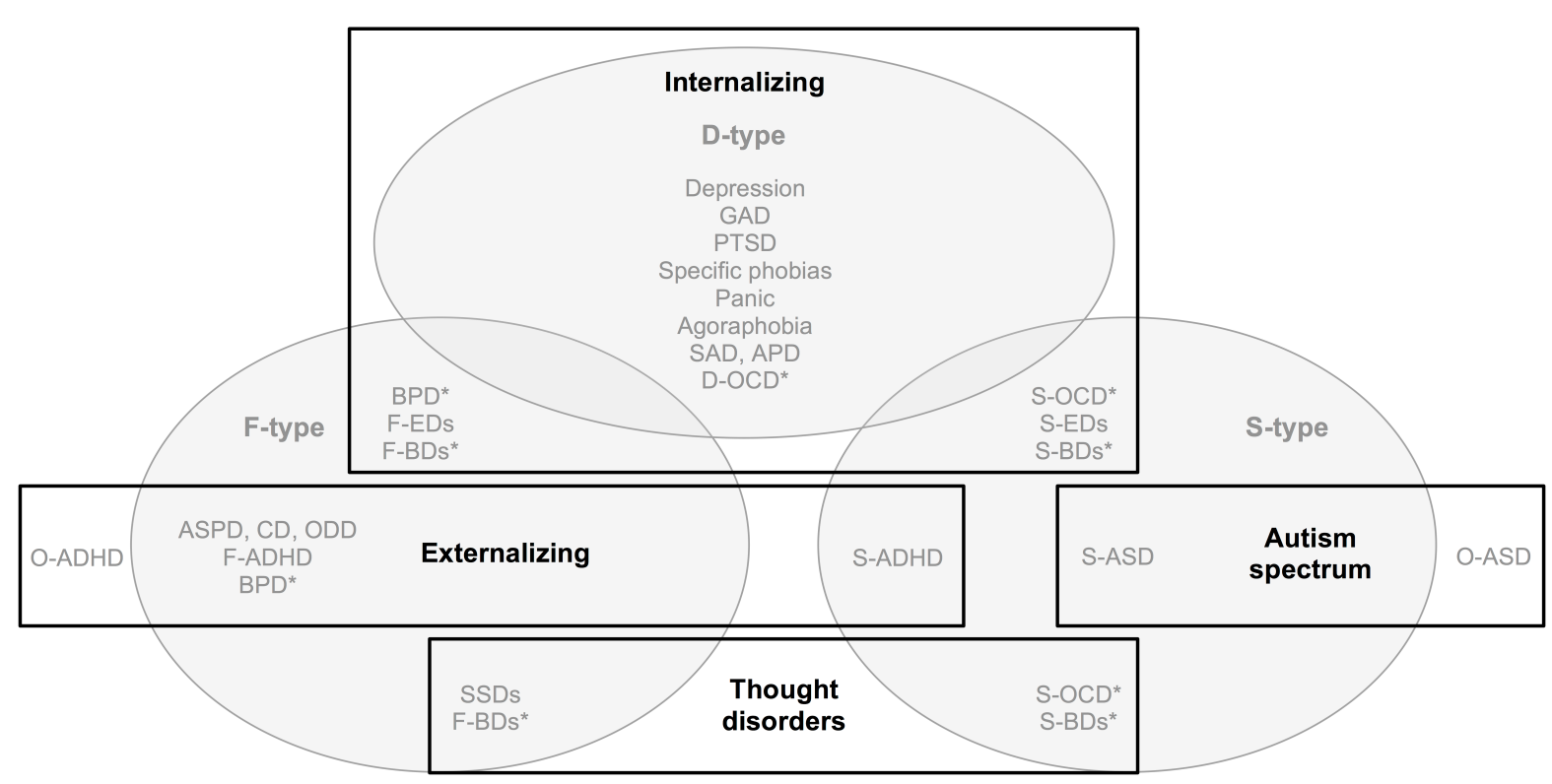

Figure 3. Overlap and differences between the FSD classification and the core dimensions of transdiagnostic models. Asterisks denote conditions that are regarded as "interstitial", or have been assigned to different spectra by different authors. See Figure 2 for acronyms. Reproduced with permission from Del Giudice (2018).

Figure 3 illustrates both the overlap and the differences between the FSD categories and three core dimensions of transdiagnostic models - externalizing, internalizing, and thought disorder. In addition, the HiTOP distinguishes between "disinhibited" and "antagonistic" externalizing symptoms and includes a detachment and a somatoform spectrum (Kotov et al., 2017). Autism still lacks a place in the HiTOP taxonomy, although this is an active area of investigation (e.g., Rodriguez-Seijas et al., 2020). As can be seen from the figure, the defense activation (D-type) category overlaps to a large extent with the internalizing spectrum, and maintains the heuristic distinction between "fear" and "distress" disorders (see Kotov et al., 2017). At the same time, there are important differences: most notably, eating disorders (which are especially hard to square with the internalizing-externalizing distinction) are split between the fast and slow spectra, BPD is classified as an F-type condition, and OCD includes an S-type 
variant. Whereas the HiTOP locates APD symptoms within the detachment spectrum (which in turn is nested within a psychosis superspectrum; Kotov et al., 2020), the FSD model regards APD as a more severe variant of SAD and includes it in the defense activation cluster. Similar considerations can be made with respect to externalizing conditions. Finally, the FSD model does not have a separate classification for thought disorders, and the relevant conditions are split between the fast and slow spectra (plus the D-type variant of OCD, which is regarded as a disorder of hyperactivated harm prevention mechanisms).

\section{The Meaning of the "p Factor"}

The basic dimensions described by the HiTOP and related transdiagnostic models (internalizing, externalizing, thought disorder, and so forth) are not independent, but instead show a pattern of positive correlations. By fitting factor-analytic models to the data, it is possible to extract a generalized factor of psychopathology that cuts across symptom dimensions - the socalled "p factor" (Caspi \& Moffitt, 2018; Caspi et al., 2014, 2020; Lahey et al., 2017a, 2017b). The HiTOP provisionally includes the $\mathrm{p}$ factor as a higher-order dimension in the upper stratum of the taxonomy (Conway et al., 2019; Krueger et al., 2018). The p factor is about 50\% heritable; across a range of developmental periods and in both community and clinical cohorts, higher scores on the $\mathrm{p}$ factor have been correlated with high neuroticism, low agreeableness (antagonism), low conscientiousness, impulsivity, intellective deficits/low IQ, reduced neural integrity (e.g., white matter microstructure), and early adversity and trauma (Allegrini et al., 2020; Caspi \& Moffitt, 2018; Caspi et al., 2020). Additionally, higher scores on the p factor have been associated with self-harm and suicidal ideation (Haltigan et al., 2018).

Many researchers view the $\mathrm{p}$ factor as a broad, nonspecific liability that increases the risk for mental disorders across the board (see Caspi \& Moffitt, 2018; Conway et al., 2019). The framework we have presented suggests a different interpretation. From the standpoint of the FSD model, the seemingly unitary $\mathrm{p}$ factor is a heterogeneous construct, arising from a mixture of three largely independent functional components: fast life history strategies (reflected in impulsivity and antagonism), (b) generalized activation of defensive mechanisms (reflected in elevated neuroticism), and (c) low cognitive ability (Del Giudice, 2018). Interestingly, Caspi and Moffitt (2018) discussed negative affect, poor impulse control, and cognitive deficits as three alternative hypotheses about the nature of the $\mathrm{p}$ factor; the FSD model views them as markers of three fundamental dimensions of individual variation that affect the risk of psychopathology through specific etiological pathways. A unique prediction stemming from this perspective is that, since the $\mathrm{p}$ factor partly reflects a dimension of fast life history strategy, slow spectrum disorders (such as high-functioning autism, OCPD, and certain subtypes of eating disorders and OCD) should be less strongly associated with the $p$ factor than their fast spectrum counterparts (Del Giudice, 2016c). (One does not expect a simple negative association with p, because S-type disorders overlap with D-type disorders and can be exacerbated by cognitive deficits.) Of course, a functionally heterogeneous dimension can still have descriptive and clinical utility (Meehl, 1993). At the same time, unitary conceptions of the $p$ factor (e.g., as a dimension of "disordered thought"; see Caspi \& Moffitt, 2018) may lead researchers to blur important boundaries, and ascribe distinct etiological processes to the same nonspecific source. 
The idea of $\mathrm{p}$ as a radically heterogeneous construct was supported by a simulation study in which symptom scores were generated according to an early version of the FSD model, but analyzed with the same factor-analytic models employed in empirical studies of the $p$ factor (Del Giudice, 2016c). As predicted, standard factor-analytic techniques recovered distinct internalizing, externalizing, and thought disorder factors, as well as a general $\mathrm{p}$ factor-none of which figured in the true model used to generate the data. There are two main reasons for these surprising findings. First, transdiagnostic models such as the HiTOP currently lack the notion of functional subtypes; because transdiagnostic dimensions such as "internalizing" and "externalizing" are based on symptom correlations, they cannot be used to distinguish between similar constellations of symptom that arise for functionally different reasons. Second, the factoranalytic models that are most commonly employed in this area assume linear relations between factors, and hence are unable to recover nonlinear relations between constructs (e.g., the risk for defense activation disorders increases at both ends of the fast-slow continuum). While these results do not prove that the FSD model is correct, they do cast doubt on a unitary interpretation of the $p$ factor. Similarly, Watts et al. (2019) concluded that the $p$ factor is more likely to reflect an "amalgam of psychopathology" rather than a generalized liability. The life history framework goes one step further by offering a functional interpretation of the main components summarized by the $\mathrm{p}$ factor.

\section{Conclusion}

Meaningful progress in the study of psychopathology will require a combination of highquality empirical data and well-grounded theory. Transdiagnostic models such as the HiTOP offer a parsimonious description of the structure of mental disorders, but so far have not been able to move from empirical generalizations to a genuine theoretical framework for the discipline (Dalgeish et al., 2020). Other theoretical approaches highlight important functional principles, but are too narrow to answer outstanding questions about comorbidity, heterogeneity, individual differences, and development. We concur with Brüne et al. (2012) that a coherent understanding of psychopathology will remain elusive until researchers adopt a thoroughly evolutionary view of the human mind/brain and its disorders. From this broad metatheoretical perspective, we presented a life history framework for the analysis of mental disorders and a taxonomy based on its concepts, the FSD model (Del Giudice, 2018). To be sure, additional work is needed to test the empirical predictions of the model, extend its coverage, and refine it from a theoretical standpoint. Nonetheless, we believe that this approach has already shown considerable potential and heuristic value. We hope that researchers in the field will find it exciting as we do, and begin considering it as a source of ideas for empirical studies, as well as a promising candidate for integration with other theoretical models. 


\section{References}

Allegrini, A. G., Cheesman, R., Rimfeld, K., Selzam, S., Pingault, J. B., Eley, T. C., \& Plomin, R. (2020). The $\mathrm{p}$ factor: Genetic analyses support a general dimension of psychopathology in childhood and adolescence. Journal of Child Psychology and Psychiatry, 61, 30-39.

American Psychiatric Association (2013). Diagnostic and Statistical Manual of mental disorders ( $5^{\text {th }}$ ed.). APA.

Ashton, M. C., \& Lee, K. (2008). The HEXACO model of personality structure and the importance of the H factor. Social and Personality Psychology Compass, 2, 1952-1962.

Belsky, J., \& Pluess, M. (2016). Differential susceptibility to environmental influences. In D. Cicchetti (Ed.), Developmental Psychopathology (3 ${ }^{\text {rd }}$ ed.), Vol. 2: Developmental Neuroscience (pp. 59-106). Wiley.

Belsky, J., Steinberg, L., \& Draper, P. (1991). Childhood experience, interpersonal development, and reproductive strategy: An evolutionary theory of socialization. Child Development, 62, 647-670.

Borsboom, D., Cramer, A. O., \& Kalis, A. (2019). Brain disorders? Not really: Why network structures block reductionism in psychopathology research. Behavioral and Brain Sciences, 42, e2.

Borsboom, D., \& Cramer, A. O. (2013). Network analysis: An integrative approach to the structure of psychopathology. Annual Review of Clinical Psychology, 9, 91-121.

Braendle, C., Heyland, F., \& Flatt, T. (2011). Integrating mechanistic and evolutionary analysis of life history variation. In T. Flatt \& F. Heyland (Eds.), Mechanisms of life history evolution. The genetics and physiology of life history traits and trade-offs (pp. 3-10). Oxford University Press.

Brüne, M. (2015). Textbook of evolutionary psychiatry and psychosomatic medicine: The origins of psychopathology. Oxford University Press.

Brüne, M., Belsky, J., Fabrega, H., Feierman, H. R., Gilbert, P., Glantz, K., ... \& Troisi, A. (2012). The crisis of psychiatry-insights and prospects from evolutionary theory. World Psychiatry, 11, 55-57.

Brüne, M., Ghiassi, V., \& Ribbert, H. (2010). Does borderline personality reflect the pathological extreme of an adaptive reproductive strategy? Insights and hypotheses from evolutionary life-history theory. Clinical Neuropsychiatry, 7, 3-9.

Brüne, M., \& Schiefenhovel, W. (Eds.) (2019). Oxford Handbook of Evolutionary Medicine. Oxford University Press.

Buss, D. M. (Ed.). (2015). The handbook of evolutionary psychology (2 ${ }^{\text {nd }}$ ed.). Wiley.

Caspi, A., Houts, R. M., Ambler, A., Danese, A., Elliott, M. L., Ariri, A., ... \& Moffitt, T. E. (2020). Longitudinal assessment of mental health disorders and comorbidities across 4 decades among participants in the Dunedin Birth Cohort Study. JAMA Network Open, 3, e203221.

Caspi, A., Houts, R. M., Belsky, D. W., Goldman-Mellor, S. J., Harrington, H., Israel, S., ... \& Moffitt, T. E. (2014). The p factor: One general psychopathology factor in the structure of psychiatric disorders? Clinical Psychological Science, 2, 119-137. 
Caspi, A., \& Moffitt, T. E. (2018). All for one and one for all: Mental disorders in one dimension. American Journal of Psychiatry, 175, 831-844.

Coghill, D., \& Sonuga-Barke, E. J. (2012). Categories versus dimensions in the classification and conceptualisation of child and adolescent mental disorder: Implications of recent empirical study. Journal of Child Psychology and Psychiatry, 53, 469-489.

Conway, C. C., Forbes, M. K., Forbush, K. T., Fried, E. I., Hallquist, M. N., Kotov, R., ... \& Sunderland, M. (2019). A hierarchical taxonomy of psychopathology can transform mental health research. Perspectives on Psychological Science, 14, 419-436.

Cosmides, L., \& Tooby, J. (1999). Toward an evolutionary taxonomy of treatable conditions. Journal of Abnormal Psychology, 108, 453-464.

Crespi, B. J. (2010). The origins and evolution of genetic disease risk in modern humans. Annals of the New York Academy of Sciences, 1206, 80-109.

Crespi, B. J. (2011). One hundred years of insanity: Genomic, psychological, and evolutionary models of autism in relation to schizophrenia. In M. S. Ritsner (Ed.), Handbook of schizophrenia spectrum disorders (Vol. I., pp. 163-185). Springer.

Crespi, B. J. (2019). Autism, psychosis, and genomic imprinting: Recent discoveries and conundrums. Current Opinion in Behavioral Sciences, 25, 1-7.

Crespi, B. J. (2020). Evolutionary and genetic insights for clinical psychology. Clinical Psychology Review, 78, 101857.

Crespi, B., \& Badcock, C. (2008). Psychosis and autism as diametrical disorders of the social brain. Behavioral and Brain Sciences, 31, 241-261.

Cuthbert, B. N., \& Insel, T. R. (2013). Toward the future of psychiatric diagnosis: The seven pillars of RDoC. BMC Medicine, 11, 126.

Dalgleish, T., Black, M., Johnston, D., \& Bevan, A. (2020). Transdiagnostic approaches to mental health problems: Current status and future directions. Journal of Consulting and Clinical Psychology, 88, 179-195.

Del Giudice, M. (2012). Sex ratio dynamics and fluctuating selection on personality. Journal of Theoretical Biology, 297, 48-60.

Del Giudice, M. (2014). An evolutionary life history framework for psychopathology. Psychological Inquiry, 25, 261-300.

Del Giudice, M. (2016a). The evolutionary future of psychopathology. Current Opinion in Psychology, 7, 44-50.

Del Giudice, M. (2016b). Differential susceptibility to the environment: Are developmental models compatible with the evidence from twin studies? Developmental Psychology, 52, 1330-1339.

Del Giudice, M. (2016c). The life history model of psychopathology explains the structure of psychiatric disorders and the emergence of the p factor: A simulation study. Clinical Psychological Science, 4, 299-311.

Del Giudice, M. (2017). Mating, sexual selection, and the evolution of schizophrenia. World Psychiatry, 16, 141-142.

Del Giudice, M. (2018). Evolutionary psychopathology: A unified approach. Oxford University Press. 
Del Giudice, M. (2020). Rethinking the fast-slow continuum of individual differences. Evolution and Human Behavior.

Del Giudice, M. (2021). Are we comparing apples or squared apples? The proportion of explained variance exaggerates differences between effects. PsyArxiv, https://doi.org/10.31234/osf.io/bgh54

Del Giudice, M., Angeleri, R., Brizio, A., \& Elena, M. R. (2010). The evolution of autistic-like and schizotypal traits: A sexual selection hypothesis. Frontiers in Psychology, 1, 41.

Del Giudice, M., Gangestad, S. W., \& Kaplan, H. S. (2015). Life history theory and evolutionary psychology. In D. M. Buss (Ed.), The handbook of evolutionary psychology $\left(2^{\text {nd }} \mathrm{ed}\right.$., pp. 88-114). Wiley.

Del Giudice, M., Klimczuk, A. C. E., Traficonte, D. M., \& Maestripieri, D. (2014). Autistic-like and schizotypal traits in a life history perspective: Diametrical associations with impulsivity, sensation seeking, and sociosexual behavior. Evolution \& Human Behavior, $35,415-424$.

DeYoung, C. G., \& Krueger, R. F. (2018). A cybernetic theory of psychopathology. Psychological Inquiry, 29, 117-138.

Durisko, Z., Mulsant, B. H., McKenzie, K., \& Andrews, P. W. (2016). Using evolutionary theory to guide mental health research. Canadian Journal of Psychiatry, 61, 159-165.

Ellis, B. J., \& Del Giudice, M. (2019). Developmental adaptation to stress: An evolutionary perspective. Annual Review of Psychology, 70, 111-139.

Ellis, B. J., Figueredo, A. J., Brumbach, B. H., \& Schlomer, G. L. (2009). The impact of harsh versus unpredictable environments on the evolution and development of life history strategies. Human Nature, 20, 204-268.

Fraley, R. C., Roisman, G. I., \& Haltigan, J. D. (2013). The legacy of early experiences in development: Formalizing alternative models of how early experiences are carried forward over time. Developmental Psychology, 49, 109-126.

Galipaud, M., \& Kokko, H. (2020). Adaptation and plasticity in life-history theory: How to derive predictions. Evolution and Human Behavior, 41, 493-501.

Grove, J., Ripke, S., Als, T. D., Mattheisen, M., Walters, R. K., Won, H., ... \& Awashti, S. (2019). Identification of common genetic risk variants for autism spectrum disorder. Nature Genetics, 51, 431-444.

Haeffel, G. J., Jeronimus, B. F., Kaiser, B. N., Weaver, L. J., Soyster, P. D., Fisher, A. J., ... \& Lu, W. (2021). Folk classification and factor rotations: Whales, sharks, and the problems with the Hierarchical Taxonomy of Psychopathology (HiTOP). Clinical Psychological Science. doi:21677026211002500.

Haltigan, J. D., Aitken, M. A., Skilling, T., Henderson, J., Hawke, L., Battaglia, M., Strauss, J., Szatmari, P., Andrade, B. F. (2018). "P" and "DP:" Examining Symptom-Level Bifactor Models of Psychopathology and Dysregulation in Clinically Referred Children and Adolescents. Journal of the American Academy of Child and Adolescent Psychiatry, 57, 384-396. doi: https://doi.org/10.1016/j.jaac.2018.03.010.

Haslam, N., Holland, E., \& Kuppens, P. (2012). Categories versus dimensions in personality and psychopathology: A quantitative review of taxometric research. Psychological Medicine, 42, 903-920. 
Haslam, N., McGrath, M. J., Viechtbauer, W., \& Kuppens, P. (2020). Dimensions over categories: A meta-analysis of taxometric research. Psychological Medicine, https://doi.org/10.1017/S003329172000183X

Healy, K., Ezard, T. H., Jones, O. R., Salguero-Gómez, R., \& Buckley, Y. M. (2019). Animal life history is shaped by the pace of life and the distribution of age-specific mortality and reproduction. Nature Ecology \& Evolution, doi: 10.1038/s41559-019-0938-7

Hilbert, A., Pike, K. M., Wilfley, D. E., Fairburn, C. G., Dohm, F. A., \& Striegel-Moore, R. H. (2011). Clarifying boundaries of binge eating disorder and psychiatric comorbidity: A latent structure analysis. Behaviour research and therapy, 49, 202-211.

Hunt, J., \& Hodgson, D. (2010). What is fitness, and how do we measure it? In D. F. Westneat \& C. W. Fox (Eds.), Evolutionary behavioral ecology (pp. 46-70). Oxford University Press.

Huys, Q. J., Guitart-Masip, M., Dolan, R. J., \& Dayan, P. (2015). Decision-theoretic psychiatry. Clinical Psychological Science, 3, 400-421.

Keller, M. C. (2018). Evolutionary perspectives on genetic and environmental risk factors for psychiatric disorders. Annual Review of Clinical Psychology, 14, 471-493.

Kendler, K. S. (2018). Classification of psychopathology: Conceptual and historical background. World Psychiatry, 17, 241-242.

Kendler, K. S., Ohlsson, H., Lichtenstein, P., Sundquist, J., \& Sundquist, K. (2019). The nature of the shared environment. Behavior Genetics, 49, 1-10.

Knopik, V. S., Neiderhiser, J. M., DeFries, J. C., \& Plomin, R. (2017). Behavioral genetics $\left(7^{\text {th }}\right.$ ed.). Worth.

Kotov, R., Krueger, R. F., Watson, D., Achenbach, T. M., Althoff, R. R., Bagby, R. M., ... \& Eaton, N. R. (2017). The Hierarchical Taxonomy of Psychopathology (HiTOP): A dimensional alternative to traditional nosologies. Journal of Abnormal Psychology, 126, 454.

Krueger, R. F., Hobbs, K. A., Conway, C. C., Dick, D. M., Dretsch, M. N., Eaton, N. R., ... \& Workgroup, H. U. (2021). Validity and utility of Hierarchical Taxonomy of Psychopathology (HiTOP): II. Externalizing superspectrum. World psychiatry, 20, 171193.

Krueger, R. F., Kotov, R., Watson, D., Forbes, M. K., Eaton, N. R., Ruggero, C. J., ... \& Bagby, R. M. (2018). Progress in achieving quantitative classification of psychopathology. World Psychiatry, 17, 282-293.

Lahey, B. B., Krueger, R. F., Rathouz, P. J., Waldman, I. D., \& Zald, D. H. (2017). Validity and utility of the general factor of psychopathology. World Psychiatry, 16, 142-144.

Lahey, B. B., Krueger, R. F., Rathouz, P. J., Waldman, I. D., \& Zald, D. H. (2017). A hierarchical causal taxonomy of psychopathology across the life span. Psychological Bulletin, 143, 142-186.

Larsson, H., Ryden, E., Boman, M., Långström, N., Lichtenstein, P., \& Landen, M. (2013). Risk of bipolar disorder and schizophrenia in relatives of people with attention-deficit hyperactivity disorder. British Journal of Psychiatry, 203, 103-106.

Lawn, R. B., Sallis, H. M., Taylor, A. E., Wootton, R. E., Smith, G. D., Davies, N. M., ... \& Munafo, M. R. (2019). Schizophrenia risk and reproductive success: A Mendelian randomization study. Royal Society Open Science, 6, 181049. 
Lee, P. H., Anttila, V., Won, H., Feng, Y. C. A., Rosenthal, J., Zhu, Z., ... \& Wang, M. M. J. (2019). Genomic relationships, novel loci, and pleiotropic mechanisms across eight psychiatric disorders. Cell, 179, 1469-1482.

Linnér, R. K., Biroli, P., Kong, E., Meddens, S. F. W., Wedow, R., Fontana, M. A., ... \& Okbay, A. (2018). Genome-wide study identifies 611 loci associated with risk tolerance and risky behaviors. BioRxiv, 261081.

Linner, R. K., Mallard, T. T., Barr, P. B., Sanchez-Roige, S., Madole, J. W., Driver, M. N., ... \& Dick, D. M. (2020). Multivariate genomic analysis of 1.5 million people identifies genes related to addiction, antisocial behavior, and health. BioRxiv, 342501.

McGuire, M. T., \& Troisi, A. (1998). Darwinian psychiatry. Oxford University Press.

Mealey, L. (1995). The sociobiology of sociopathy: An integrated evolutionary model. Behavioral and Brain Sciences, 18, 523-541.

Meehl, P. E. (1993). Four queries about factor reality. History and Philosophy of Psychology Bulletin, 5, 4-5.

Musser, E. D., Hawkey, E., Kachan-Liu, S. S., Lees, P., Roullet, J. B., Goddard, K., ... \& Nigg, J. T. (2014). Shared familial transmission of autism spectrum and attentiondeficit/hyperactivity disorders. Journal of Child Psychology and Psychiatry, 55, 819-827.

Nesse, R. M. (2005b). Maladaptation and natural selection. The Quarterly Review of Biology, 80, $62-70$.

Nesse, R. M. (2019). Good reasons for bad feelings: Insights from the frontier of evolutionary psychiatry. Penguin.

Nesse, R. M., \& Jackson, E. D. (2006). Evolution: Psychiatric nosology's missing biological foundation. Clinical Neuropsychiatry, 3, 121-131.

Nettle, D. (2001). Strong imagination: Madness, creativity and human nature. Oxford University Press.

Nettle, D., \& Frankenhuis, W. E. (2019). The evolution of life-history theory: A bibliometric analysis of an interdisciplinary research area. Proceedings of the Royal Society of London $B, 286,20190040$.

Nettle, D., \& Frankenhuis, W. E. (2020). Life history theory in psychology and evolutionary biology: One research programme or two? Philosophical Transactions of the Royal Society B. https://doi.org/10.1098/rstb.2019.0490

Ni, G., Amare, A. T., Zhou, X., Mills, N., Gratten, J., \& Lee, S. H. (2019). The genetic relationship between female reproductive traits and six psychiatric disorders. Scientific Reports, 9, 12041.

Rhee, S. H., \& Waldman, I. D. (2002). Genetic and environmental influences on antisocial behavior: A meta-analysis of twin and adoption studies. Psychological Bulletin, 128, 490529.

Rodriguez-Seijas, C., Gadow, K. D., Rosen, T. E., Kim, H., Lerner, M. D., \& Eaton, N. R. (2020). A transdiagnostic model of psychiatric symptom co-occurrence and autism spectrum disorder. Autism Research, 13, 579-590.

Roff, D. A. (2002). Life history evolution. Sinauer.

Salmon, C., Figueredo, A. J., \& Woodburn, L. (2009). Life history strategy and disordered eating behavior. Evolutionary Psychology, 7, 585-600. 
Seery, M. D. (2011). Resilience: A silver lining to experiencing adverse life events? Current Directions in Psychological Science, 20, 390-394.

Seery, M. D., Leo, R. J., Lupien, S. P., Kondrak, C. L., \& Almonte, J. L. (2013). An upside to adversity? Moderate cumulative lifetime adversity is associated with resilient responses in the face of controlled stressors. Psychological Science, 24, 1181-1189.

Selzam, S., Coleman, J. R., Caspi, A., Moffitt, T. E., \& Plomin, R. (2018). A polygenic p factor for major psychiatric disorders. Translational Psychiatry, 8, 205.

Seth, A. K., \& Friston, K. J. (2016). Active interoceptive inference and the emotional brain. Philosophical Transactions of the Royal Society B, 371, 20160007.

Shaner, A., Miller, G. F., \& Mintz, J. (2004). Schizophrenia as one extreme of a sexually selected fitness indicator. Schizophrenia Research, 70, 101-109.

Solberg, B. S., Zayats, T., Posserud, M. B., Halmøy, A., Engeland, A., Haavik, J., \& Klungsøyr, K. (2019). Patterns of psychiatric comorbidity and genetic correlations provide new insights into differences between attention-deficit/hyperactivity disorder and autism spectrum disorder. Biological Psychiatry, 86, 587-598.

Stearns, S. C. (1992). The evolution of life histories. Oxford University Press.

Stearns, S. C., \& Medzhitov, R. (2016). Evolutionary medicine. Sinauer.

Syme, K. L., \& Hagen, E. H. (2019). Mental health is biological health: Why tackling "diseases of the mind" is an imperative for biological anthropology in the 21 st century. American Journal of Physical Anthropology, https://doi.org/10.1002/ajpa.23965

Thomas, J. J., Eddy, K. T., Ruscio, J., Ng, K. L., Casale, K. E., Becker, A. E., \& Lee, S. (2015). Do recognizable lifetime eating disorder phenotypes naturally occur in a culturally Asian population? A combined latent profile and taxometric approach. European Eating Disorders Review, 23, 199-209.

Vitousek, M. N., \& Schoenle, L. A. (2019). Hormones and behavior: A life history perspective. In L. M. Welling \& T. K. Shackelford (Eds.), The Oxford handbook of evolutionary psychology and behavioral endocrinology. Oxford University Press.

Voon, V., Reiter, A., Sebold, M., \& Groman, S. (2017). Model-based control in dimensional psychiatry. Biological Psychiatry, 82, 391-400.

Wakefield, J. C. (1992). The concept of mental disorder: On the boundary between biological facts and social values. American Psychologist, 47, 373-388.

Wakefield, J. C. (1999a). Evolutionary versus prototype analyses of the concept of disorder. Journal of Abnormal Psychology, 108, 374-399.

Wakefield, J. C. (2014). Wittgenstein's nightmare: Why the RDoC grid needs a conceptual dimension. World Psychiatry, 13, 38-40.

Warrier, V., Toro, R., Won, H., Leblond, C. S., Cliquet, F., Delorme, R., ... \& Grove, J. (2019). Social and non-social autism symptoms and trait domains are genetically dissociable. Communications Biology, 2, 328.

Watts, A. L., Poore, H. E., \& Waldman, I. D. (2019). Riskier tests of the validity of the bifactor model of psychopathology. Clinical Psychological Science, 7, 1285-1303.

West, S. A., \& Gardner, A. (2013). Adaptation and inclusive fitness. Current Biology, 23, r577r584. 
Williamson, D. A., Gleaves, D. H., \& Stewart, T. M. (2005). Categorical versus dimensional models of eating disorders: An examination of the evidence. International Journal of Eating Disorders, 37, 1-10.

Wright, J., Bolstad, G. H., Araya-Ajoy, Y. G., \& Dingemanse, N. J. (2019). Life-history evolution under fluctuating density-dependent selection and the adaptive alignment of pace-of-life syndromes. Biological Reviews, 94, 230-247.

Zhang, Y., Lu, Q., Ye, Y., Huang, K., Liu, W., Wu, Y., ... \& Werling, D. (2020). Local genetic correlation analysis reveals heterogeneous etiologic sharing of complex traits. bioRxiv, doi: https://doi.org/10.1101/2020.05.08.084475

Zheng, Y., Kang, Q., Huang, J., Jiang, W., Liu, Q., Chen, H., .. \& Chen, J. (2019). The classification of eating disorders in China: A categorical model or a dimensional model. International Journal of Eating Disorders, 52, 712-720.

Zietsch, B. P., \& Sidari, M. J. (2020). A critique of life history approaches to human trait covariation. Evolution and Human Behavior, doi: 10.1016/j.evolhumbehav.2019.05.007 